\title{
The Analysis of Korean Entertainment Companies' Business Strategy in The Greater China from the view of Value Chain -Exemplified by S. M. Entertainment and EXO
}

\author{
Yuanchao Liu \\ National Taiwan Normal University, Taipei, Taiwan, China \\ 323151451@qq.com
}

Keywords: K-POP, Value Chain, SME, Overseas Strategy, Brand Management

\begin{abstract}
Under the background of globalization and digital convergence, the media integration and industrial transformation are the new outlets for the entertainment industry in the wave of future. With its extraordinary integration of upstream and downstream industries, S.M.Entertainment from Korea has become one of the most representative and indicative entertainment companies in today's Asian entertainment industry, a model of cross cutting and diversified development. This research uses the structure of value chain theory as basis and categorizes the activities of corporation value through the marketing behavior and strategy implementation of SME. Furthermore, it provides in-depth analysis of business empire of SME in terms of operation of overseas business strategy and establishment of brand image.

The research takes top male group EXO created by the leading representative of K-POP S.M.Entertainment as example to determine the best approach for the global expansion of new infotainment media industry in the context of industry convergence. The primary methodology includes secondary data analysis and case study focusing on four aspects as research framework including early-stage preparation, content production, research and marketing, and value recovery. In the case study, SME firstly establish its unique and thorough work flow and operating system, and concentrate on New Culture Technology's globalization strategy. In addition, SME has huge investment in every stage of cultural commodity production to continuously improve the quality of content meanwhile utilizing new digital technology to strengthen their competitiveness in Asia and the global market. During the ongoing fight of SME for Chinese market share, the researcher who based on the cross-strait hopes the insight of SME research can deliver the unique strategies of SME on local basis to the workers in the field of the Greater China entertainment industry.
\end{abstract}

\section{从价值链看韩国娱乐公司在大中华地区的经营策略 \\ 一以 S. M. Entertainment 及旗下团体 EXO 为例}

\author{
刘远超1,a \\ 国立台湾师范大学, 台北, 台湾, 中国
}

323151451@qq.com

刘远超

关键词: K-POP; 价值链; SME; 海外策略; 品牌经营

中文摘要. 在全球化和数位汇流的大背景下, 媒介融合和产业转型是娱乐文化产业在大势所 趋下纷纷寻求的新出路。凭藉着对产业出色的上下游垂直整合能力, 韩国的SME无疑成为了 当今亚洲娱乐产业中最具代表性的指标型娱乐经济公司。本研究以价值链理论的架构作为基 
础, 透过SME的行销行为和策略实施来进行企业价值活动的划分, 深入解析SME这一庞大的 商业帝国究竟是如何运营它的海外经营策略以及树立品牌形象。

本研究借镜K-POP领军代表S.M.Entertainment打造登顶男团EXO的案例, 找出新型资讯娱乐媒 体产业因应产业汇流背景扩展全球化策略的最佳方式。主要运用次级资料分析法和案例分析 法, 借由前期准备, 内容产制, 研发与行销, 以及价值回收四个层面作为研究架构。

从SME的案例研究中, 发现SME首先建立其独特而完善的作业流程和运营体系, 围绕着New Culture Technology的全球化策略, 在文化商品产制的每一个阶段都投入十足的成本不断提高 内容品质, 运用新型数位科技强化自身在亚洲乃至全球的市场竞争力。在SME争夺华语市场 份额的进程中, 研究者立足两岸, 希望可以透过对SME的研究, 将其策略之独到, 因地制宜 地提供给大中华地区娱乐业者参详。

\section{1. 研究背景}

近年来, 韩国靠着其独特的文化产业高速发展软实力, 将韩国文化推向世界舞台, 在世 界各地，尤其是亚洲地区形成了以“哈韩族”为代表的迷群文化。韩国流行文化借Korea-Pop(韩 国流行音乐, 简称K-POP)强势入侵, 通过对偶像团体整合包装, 集体行销的方式, 借助大众 媒介的即时传播力量迅速占领亚洲甚至世界市场，并带动其周边产品形成强大的粉丝经济。 靠着成功的偶像团体营销手法, 将“韩流”的中心从韩国电视剧所创造出的偶像崇拜, 进而转 向记忆点更加强烈的K-POP。由于韩国本国文化产品消费市场较为狭小, 因此决定了韩国的 流行文化只有向外输出才能谋求更大的经济效益。而亚洲范围内人口数居世界第一且购买潜 力巨大的潜在市场一一中华地区则成为了韩流输出的首选考量对象。大中华地区泛指“大中 华经济圈”在上世纪八十年代初被提出, 是指中国内地、香港、澳门、台湾完全实现自由贸易 区。根据美国《商业周刊》对“大中华经济圈”的研究显示: “『大中华经济圈』在世界出口贸 易中的比重由 $6.9 \%$ 上升到 $9.6 \%$, 已超过日本。”大中华区无疑已经成为K-POP在新时期对外 输出的首要战略目标, 得中国大陆更是等于得到整个华语市场。整个大中华区相对应的市场 经济, 对于韩国而言具有较强的历史和文化近源性, 对文化产品的接受度相对于其他地区更 高, 因此成为韩国娱乐文化输出海外的头号战略目标。

SME作为韩国流行音乐产业的领军集团, 其在公司品牌塑造方面取得了有目共睹的卓越 成绩，同时也为韩国流行音乐在世界范围内的推广起到了巨大推动作用。SME以独到的视角 和长远的目光, 利用市场区隔, 将市场进行进一步的细分, 从而选定相应的最适用的行销方 式在其目标市场中进行推广行销, 使企业策略可以随着市场的变化进行调整, 紧跟上市场的 变化以及阅听众的偏好。如何在竞争激烈, 群雄逐鹿的华语音乐市场占得先机, 成功地建立 其品牌形象并永续经营是为一个具有研究和分析意义的课题。

\section{2. 研究目的与价值}

随着韩国流行文化进一步走出国门, 韩流的影响力持续扩大, 有力带动了相关产业发展, 为韩国带来庞大的经济效益。韩国文化产业交流财团于2016年的资料显示: 韩流带来的经济 效益近年来持续增加, 2012年为 13.06 万亿韩元, 2013年为 13.83 万亿韩元, 2014 年为 14.29 万 亿韩元。而 2015 年韩流催生的经济效益高达 15.61 万亿韩元（约合人民币 878 亿元），同比增 长9.2\%。韩国国际交流财团发布的《2015世界韩流现状》显示: “海外韩流迷群数量已超过 3500 万。”这样势头强劲的流行文化, 势必带动背后众多产业的发展, 甚至是整个经济体的经济增 长。本研究将围绕着K-POP在亚洲市场的动向, 以及SME在大中华地区进行的行销行为进行 观察从而进行研究和分析, 一探SME系统性极强的价值活动背后的海外经营策略和品牌形象 塑造之手法以及 $S M E$ 是如何成功打入华语地区流行音乐市场的? 
在现有的研究和文献资料中, 虽有诸多以SME作为案例进行的策略分析, 但是却都只是 单一层面的讨论, 对于韩国娱乐经纪公司的研究大多集中在对于商业运行模式的研究, 而对 娱乐经纪公司的海外行销和品牌经营的研究较少。涉及文化娱乐产业的品牌研究也多以其在 韩国本土活动的案例为主, 很少涉及企业海外市场的经营。而对于SME旗下艺人团体的研究, 主要集中在对其“产品”打造和包装方式分析, 而没有将其看成整个K-POP产业的环节来进行 商业模式探讨。通过SME既成之传播效果, 研究其传播方式（策略和模式）, 才能更好地将 SME的成功要素融会贯通地掌握从而达到实际应用之目的。

\section{3. 理论基础：价值链之定义与音乐产业价值链}

Michael Porter（1985）在《竞争优势》中提出“价值链（Value chain）”的概念，认为企 业的竞争优势来自于内部各项的价值活动, 其竞争优势亦是企业策略的一部分, 即是将企业 的经营模式 (流程) 解构成一系列的价值创造过程, 而此价值流程的连结即是价值链。任何 一个企业都是其产品在设计、生产、销售、交货与售后服务等方面所进行的各项活动的聚合 体。Porter（1985）认为竞争优势来自于产品设计、生产、行销等多项独立活动建构而成。

价值链理论在不断地完善和丰富之后, 也跟随不同的产业类型演变出适配不同产业的分 析框架。每个企业在整体产业链中都是不可缺少的个体, 相互之间承上启下, 共同制造出呈 现在终端阅听众手中的价值, 而K-POP作为娱乐媒体产业的代表, 也相应发展出自身的价值链。

Bockstedt和Kauffman曾在2006年提出, 传统音乐产业的实体唱片无论内容的制作与产品 配销皆具有相当复杂的处理程序, 同时也牵扯为数众多参与者, 以此构成一个有自身特性的 产业链。在数位汇流背景下, 数位音乐快速崛起发展成为音乐产业的重要利润来源, 数位音 乐产业成为音乐产业价值链的重要组成部分。过去的音乐产业和数位音乐在产业融合中, 得 到充分的扩张和完善。数位技术为音乐产业提供了一个前所未有的发展机遇, 在音乐产业价 值链转变的过程中, 网络大幅度参与到新的数位音乐产业价值链中, 改变了发行, 销售的固 有方式, 带来新型的传播渠道, 也建立了新的盈利模式。

价值链理论作为分析架构可以很清晰地帮助本研究厘清SME的企业组织在实施和拓展其 海外策略和经营品牌时的思路, 更好地划分SME在这一过程中的价值活动, 最终体现企业绩 效。在前人的研究中, 鲜有利用价值链理论对娱乐产业进行分析的资料, 但是价值链相对于 韩国娱乐产业的产制模式可以很好地匹配, 从而作为框架进而分析, 因此本研究认为价值链 理论能够很好地适配本研究之内容。

根据企业价值链当中的基础活动与K-POP产业特质以及SME的企业情况相结合, 拟划分 出一系列价值活动, 本研究整理出的研究架构图, 如图1所示。

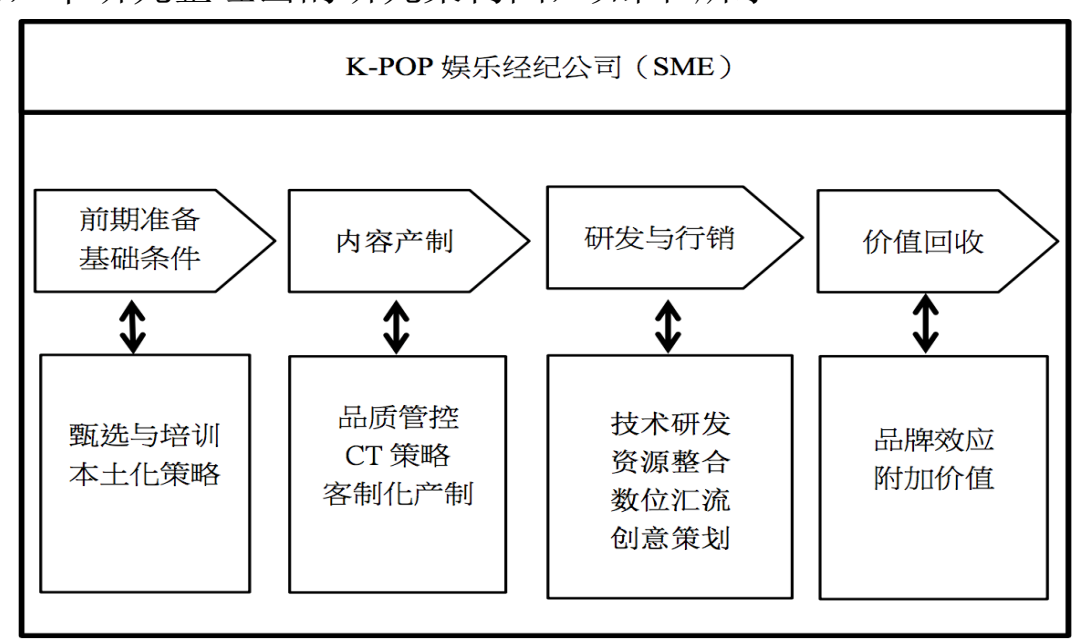

图1 研究架构 


\section{4. 研究问题}

本研究以价值链理论作为研究的框架和基础, 借由目前在亚洲市场备受瞩目的SME以及 旗下最具代表性的演艺团体EXO的案例, 旨在探讨由价值链框架下的韩国娱乐经纪公司运作 成熟的商业模式和在大中华地区为代表的海外市场之经营策略。借镜于作为亚洲娱乐业尧楚 的韩国娱乐经纪公司SME之案例, 秉持价值链的精神分析其在整个行业中脱颖而出的关键成 因，和占据大中华市场的成功经营策略。

根据研究之动机和理论架构, 本研究将拟定研究问题如下:

1.S.M.Entertainment在对外行销前的基础建设条件为何?

2. S.M.Entertainment是如何完成其在海外经营过程中的研发与行销这一重要环节的?

3.S.M.Entertainment在进入大中华区音乐市场后的价值回收表现为何?

\section{5. 研究结论}

（一）SME对外行销的基础建设条件

SME在对外行销前首先做的以严格的造星流程来完成“产品先行”，组建一个优秀的团队 共同打造一项 “文化商品”, 使其一举成为流行天团。从艺人甄选到练习生训练, 最后选拔成 员组合出道, 通过标准的SME生产线出品的偶像团体再搭配目标投放市场的本土化策略, 通 过在地化元素在目标市场率先炒热话题, 进行出道前的预热, 确保SME旗下的艺人从出道就 能获得热烈的反响。

\section{（二）SME文化商品的内容产制}

在内容产制造阶段, SME主要分成偶像作品的打造和包装, CT策略的实施, 以及客制化 产制三个环节。在这当中, 最核心的就是SME一直以来奉行的Culture Technology策略, 并在 多年的实践和摸索中, SME已经发展出适配自身特性的新型文化科技策略。在产品的打造方 面一定是下重金大手笔大制作地完成能够在市场上一鸣惊人的, 并不断寻求风格的转换和突 破，用品质过硬的音乐作品奠定偶像团体在市场的地位。同时利用创新形式的“粉丝制作人” 系统, 使文化商品更能直接满足目标客群的审美偏好, 适应海外市场, 和本土化策略更好地 融合，从而保证文化商品的输出是品质完善且匹配用户需求的“品牌商品”。

(三) SME的研发与行销

作为SME在对外输出, 争夺华语市场份额的重要步骤, 研发与行销无疑是SME整个海外 经营模式的重中之重。SME在对外行销的过程中, 利用新兴的社群媒体进行即时传播, 多元 的管道使得SME的推广企划在任何市场都可以顺利实施。同时, SME重视产业间的融合，借 由产业间的合作使SME自身加快转型发展, 更好地兼容不同产业间的资源以及并购同类型企 业达到海纳百川的效果, 将重整后拥有的上下游资源都收归旗下, 达成自身产业化经营的模 式。也正因为SME的产业化经营, 所以可以很好地自给自足, 资讯娱乐媒体产业的全部环节 都可以靠自身完成, 因此在数位汇流的潮流下可以很好地适应并且把握这一契机, 在企业的 技术研发和创意策划的发展上都更进一步，成为业界的风向标。

(四) SME传播价值的实现与回收

“产品”从产制到包装行销, SME融合整体产业中的上下游资源, 建立并完善出一套适用 自身企业的一应具全的流水线作业, 辅以在地化元素, 确保了在以内容说话的市场需求下能 以过硬的商品品质出彩, 从目标市场到潜在市场, 一举攻占华语流行音乐市场, 完成从在地 化到全球化的转变。通过在未来全球最大的市场一大中华区的成功, 成功在大中华区经营 了EXO这个品牌, 同时带动了整个SME的品牌形象。借由一系列成功的传播效果, 将EXO推 广到目标受众和潜在受众的认知中, 达成了SME在大中华区建立品牌形象和深化品牌效应的 目的。 
韩国娱乐产业，尤其是SME的成功典型对于整个华语乐坛而言是十分具有参考价值的， 可供大中华区两岸三地众多的娱乐经纪公司借镜。对比S.M.经纪公司SME成熟的商业化运作 模式, 不难发现, 首当其冲需要改进的就是建立符合自身发展需求的经纪公司机制, 系统化 的经营模式。从艺人甄选和培养, 再到内容的产制, 后期的研发行销都需要充分利用现有资 源, 下足功夫和成本, 走出最适合自己的运营规范。大中华圈经济圈, 拥有广阔的市场资源 和人力物力, 产业融合的大趋势下, 整合产业的上下游资源, 势必也能学习K-POP的成功典 范而发展出因应市场需求的娱乐经纪公司，创造大中华娱乐产业共荣的美好蓝图。

\section{References}

[1] Beck, U.[1998]. Was ist globalisierung?. Frankfurt, Germany: The Commercial Press, Ltd.)

[2] Bockstedt, J. C., Kauffman, R. J., \& Riggins, F. J. (2006). The move to artist-led on-line music distribution: a theory-based assessment and prospects for structural changes in the digital music market. International Journal of Electronic Commerce, 10(3), 7-38.

[3] Chen Yuan (2013)。New boy group EXO soaring to popularity----a miracle of South Korean star creating strategy $>$ ¿VGT WEEKLY》 No.42。

[4] Gertner,M.I.(2013).The Value Chain and Value Creation.Advances in Management,6(10)

[5] IFPI ( 2016 ) 。 《IFPI Global Music Report $2016 》 。$ Retrieved from : http://www.ifpi.org/news/IFPI-GLOBAL-MUSIC-REPORT-2016

[6] Korea Focus (2016)。The center for Korean research, Columbia University. Retrieved from : http://ckr.weai.columbia.edu/korea-focus/

[7] K-pop goes beyond Asia by internet. (2010) 。 KBS Global , Retrieved from:http://english.kbs.co.kr/hallyu/entertainment_news_view.html?

[8] Kim Yoon-mi (2011) . 《Korean Culture No.2 K-POP : A New Force in Pop music》》

[9] Kofice (2016)。《All about Korean culture》, Retrieved from : http://chn.kofice.or.kr/, http://chinese.kofice.or.kr/f00 people/f10 people.asp

[10]Kotra (2016)。Retrieved from : http://www.kotra.or.kr/kh/popup/dk ch popup.html

[11]Macintyre D. (2002). Star-studded scandal. Time, 160(5), 30-36.

[12]Morganti F. (2002).The Value Chain in Telecommunications: The growing importance of content is transforming industry structures, Intermedia,30, 5-20.

[13]Porter, M. E. (1985) . Competitive advantage: creating and sustaining superior performance: New York: The Free Press.

[14]Porter, M. E. (1991) .Toward a dynamic theory of strategy. Strategic management

[15]Philip Kotler. <Hrketing Management:Analysis, Planning, Implementation and Control $>$. New Jersey:Prentice 一 Hall, Inc, 2006 : 44-46.

[16]Pwc (2016)。 《2016 Global entertainment and media outlook》, Retrieved from : http://www.pwc.com/outlook

[17]Robbins, Stephen, P., \&Coulter, Mary (1993) .Management.Upper Saddle River,London:N.J. ; Prentice Hall International,c1999

[18]Shin, H.(2009). Have you ever seen the Rain? And who'll stop the Rain? The globalizing project of Korean pop (K-pop). Inter-Asia Cultural Studies, 10(4), 507-523 